\title{
Phenotypic diversity for quantitative and qualitative characters of barley (Hordeum vulgare) accession from Algeria
}

\author{
WARDA TAIBI ${ }^{1,2, v}$, AMINA BELLETRECHE ${ }^{1,2}$, MOHAMMED KHARSI ${ }^{3}$, \\ SEMIR BECHIR SUHEIL GAOUAR ${ }^{1,4}$, \\ ${ }^{1}$ Laboratory of Physiology, Physiopathology and Biochemistry of Nutrition (PPABIONUT), Department of Biology, Faculty of Natural and Life \\ Sciences, Earth and Universe, Abou-Bekr Belkaïd University. 22, Rue Abi Ayad Abdelkrim, Fg Pasteur B.P 119, Tlemcen 13000, Algeria. \\ email: taibi_warda@yahoo.com \\ ${ }^{2}$ Department of Agronomy, Faculty of Natural and Life Sciences, Earth and Universe, Abou-Bekr Belkaïd University. Tlemcen 13000, Algeria \\ ${ }^{3}$ National Institute for Agronomic Research (INRA). Adrar station, Algeria \\ ${ }^{4}$ Department of Biology, Faculty of Natural and Life Sciences, Earth and Universe, Abou-Bekr Belkaïd University. Tlemcen 13000, Algeria
}

Manuscript received: 17 August 2019. Revision accepted: xxx November 2019.

\begin{abstract}
Taibi W, Belletreche A, Kharsi M, Gaouar SBS. 2019. Phenotypic diversity for quantitative and qualitative characters of barley (Hordeum vulgare) accession from Algeria. Biodiversitas 20: 3794-3803. Little is known about the diversity of barley in Algeria. An inventory has been conducted in Algeria (North and South) to collect local accessions of barley (Hordeum vulgare L.). A collection of 34 traditional and exotic accessions of barley was characterized using 12 quantitative and 18 qualitative agro-morphological traits. The phenotypic diversity was determined by the Shannon-Weaver diversity index $\left(\mathrm{H}^{\prime}\right)$ at different levels (global, by type of barley and varietal name). The $\mathrm{H}^{\prime}$ estimates showed a wide phenotypic variability for different traits with $\mathrm{H}^{\prime}$ average of 0.74 and 0.53 from quantitative and qualitative characters, respectively. The results of the multiple correspondence analysis and hierarchical clustering showed a clear distinction between the different accessions. The results of this work have revealed a great phenotypic diversity of barley accessions that only partially matches the names of varieties because of the existence of homonyms and synonyms in the names given by farmers. The information gathered from this study could be used in conventional breeding programs and in situ conservation of diversity.
\end{abstract}

Keywords: Algeria, diversity, Hordeum vulgare, morphometrics measurements

\section{INTRODUCTION}

In Algeria, barley occupied in the past a very important place, more than durum and bread wheat and formed the basis for human food. The local germplasm suffered a great genetic erosion following the introduction of new performing varieties (Rahal-Bouziane et al. 2015). Landraces are still the backbone of the agricultural systems in many developing countries, mainly in marginal environments and are characterized by high genetic heterogeneity, good adaptation to local environmental conditions and low productivity (Ceccarelli 1996). In addition, locally adapted germplasm shows many interesting traits that may be used for the improvement of current cultivars (Hadjichristodoulou 1995). Thus, assessment of the genetic variability within barley landraces is fundamental for barley breeding (Bettaieb and Attias 1992).

Barley landraces exhibit variation both between and within population. Diversity within population of these barley landraces might allow them to cope with environmental stresses, which is very important for achieving yield (Zhu et al. 2014). Morphological diversity, yield components, and yield depend on the genetic potential of the varieties used and the environmental conditions (Gabillard 1983). The use of morphological traits remains the most basic method to analyze initial germplasm resources (Zeng 2015). Morphological characterization is the foundation of genetic diversity research (Chandran and Pandya 2000), it has been used to evaluate distinctness, uniformity and stability and to establish the description of a genotype in crops including barley. This method is thought to be often influenced by environmental conditions and is labor intensive also (Russell et al. 1997).

Morphological and anatomical characters are the basis for distinguishing different species of the genus Hordeum and can be associated with agricultural productivity in many ways. For example, straw length and yield components, response to various diseases, photosynthesis, time and quantity of water requirements, applied fertilizers and pesticides, and the effects of environmental stresses such as drought, soil deficiencies, and toxicity are all partly related to plant anatomy and plant morphology (Reid 1985).

Of the thousands of Hordeum accessions available in gene banks, a significant proportion is believed to be suitable for a wide range of environments (Dawson,2015). Local varieties are generally more genetically and physically diverse than official breeds (Harlan 1975). Under unfavorable conditions, landraces may yield higher yields than modern varieties, with or without inputs (Ceccarelli 1996). Similar studies have already been carried out in Algeria on other species such as wheat (Bellatreche et al. 2012, 2016; Yakoube et al. 2018), carob tree (Yassine 
and Gaouar 2016) and olive tree (Mohamed and Gaouar 2017; Boukhari et al. 2018).

\section{MATERIALS AND METHODS}

\section{Study area}

In this study, 34 populations of Algerian diploid barley landraces were used (Table 1). The collection was made in 2016 in Tlemcen, Side Belabess, Media, Ain Defla, Bechar and Adrar regions (Figure 1).

\section{Plant materials}

The word population corresponding to a given accession cultivated by a farmer describes each collected sample. Some accessions are represented by several populations. Samples used in this study consisted of 1020 entries collected from 30 individual ears of 30 different plants taken randomly in the field from the 34 Barley accession (Table 1).

\section{Traits observation}

Thirty agro-morphological traits (quantitative and qualitative) were recorded based on the international descriptors (UPOV 1988).The18 qualitative characters were: Ear attitude(EA),number of rows(NR), ear shape(ES),curvature of first segment(CFS),sterile spikelet attitude(in mid-third of ear)(ATE),media spikelet lenght of glume and its awn relative to grain(MS/G),Grain: rachilla hair type(RH), Lemma: shape of base (SB ), Awn color(BC),spiculation of inner lateral nerves of lemma(SL), disposition of lodicules(DL),kennel: color of aleurone layer(Ck), ear: shape of the extremity (ESE), alignment of articles at the middle tier of ear( zig zag ),Grain: hairiness of ventral furrow(HVF), bosse of articles at the middle tier of ear (BA),Awns: anthocyanin coloration of tips (Pig) Awn: length compared to ear(AlE).The following 12 quantitative characters were determined: Plant length(PL),stem length(SL),ear length(EL),ear length (excluding awns) (EL/excA), number of grains per ear (NG/E), awn length (AL), density of grains per ear (DG/E),ear width(EW),ear density(ED),rachis: length of first segment $(\mathrm{mm})(\mathrm{R} / \mathrm{ls})$, grain length $(\mathrm{mm})(\mathrm{GL})$, grain width $(\mathrm{mm})(\mathrm{GW})$.

\section{Data analysis}

Each character was categorized into specific class states. The 16 qualitative and 12 quantitative characters were assigned to classes based on characteristic number quoted from UPOV (Union for the Protection of New Varieties of Plants), and analyzed using the ShannonWeaver diversity index (H; Shannon and Weaver 1949) as defined by Jain et al. (1975) to calculate phenotypical variation of each accession:

$$
\mathbf{H}=-\sum_{i=1}^{\mathrm{n}} \mathbf{P i} \operatorname{Ln} \mathbf{P i}
$$

$\mathrm{H}$ was standardized by converting it to a relative phenotypic diversity index (H') after dividing it by Hmax

$$
H^{\prime}=-\sum_{i=1}^{n} \operatorname{Pi} \operatorname{Ln} P i / \operatorname{Ln}(n)
$$

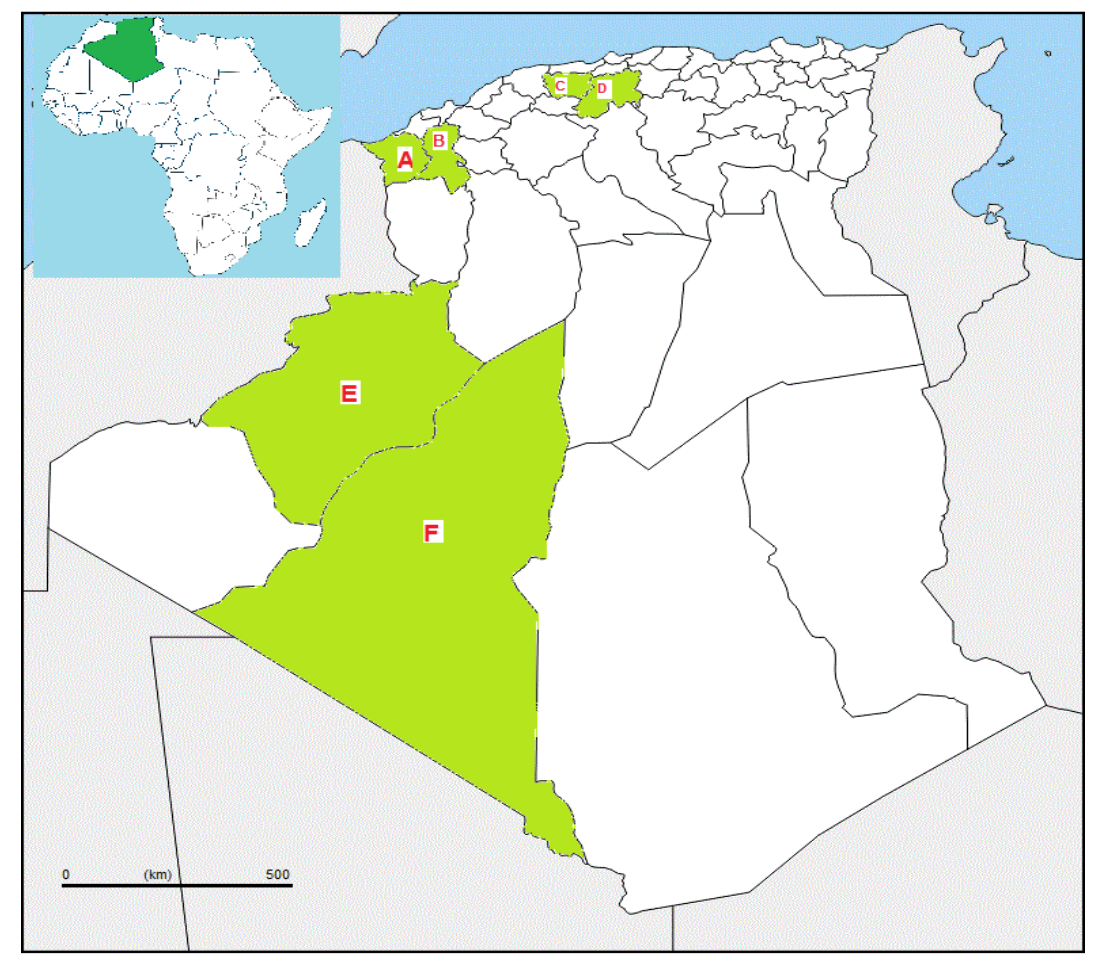


Figure 1. Location of different farmers' fields collection sites in Algeria. A. Tlemcen, B. Sidi Belabbes, C. Ain Defla, D. Media, E. Bechar and F. Adrar

Table 1. Origin of accessions studied in Algeria

\begin{tabular}{|c|c|c|c|c|c|}
\hline Accessions & Region & Locations & Longitude & Latitude & Altitude \\
\hline Saida G3 & Tlemcen & Tlemcen & $1^{\circ} 19^{\prime} 4.82^{\prime \prime O}$ & $34^{\circ} 53^{\prime} 18.26^{\prime \prime} \mathrm{N}$ & $745 m$ \\
\hline Saida G4 & & Tlemcen & $1^{\circ} 19^{\prime} 4.82^{\prime \prime O}$ & $34^{\circ} 53^{\prime} 18.26^{\prime \prime} \mathrm{N}$ & $745 \mathrm{~m}$ \\
\hline Saida R1 & & Sid Abdli & $1^{\circ} 7^{\prime} 58.47 " \mathrm{O}$ & $35^{\circ} 3^{\prime} 49.79^{\prime \prime} \mathrm{N}$ & $465 \mathrm{~m}$ \\
\hline Saida & Sidi Belabbes & Sidi Belabbes & $0^{\circ} 38^{\prime} 17.72^{\prime \prime O}$ & $35^{\circ} 12^{\prime} 8.01^{\prime \prime} \mathrm{N}$ & $478 \mathrm{~m}$ \\
\hline Saida2 & & Sidi Lahcene & $0^{\circ} 4329.18^{\prime \prime O}$ & $35^{\circ} 12^{\prime} 6.11^{\prime \prime} \mathrm{N}$ & $515 \mathrm{~m}$ \\
\hline Ascad & & Sidi Belabbes & $0^{\circ} 38^{\prime} 17.72 " \mathrm{O}$ & $35^{\circ} 12^{\prime} 7.84^{\prime \prime} \mathrm{N}$ & $478 \mathrm{~m}$ \\
\hline Rihane & & Sidi Belabbes & $0^{\circ} 38^{\prime} 17.72 " \mathrm{O}$ & $35^{\circ} 12^{\prime} 7.84^{\prime \prime N}$ & $478 \mathrm{~m}$ \\
\hline Faouara & & Sidi Belabbes & $0^{\circ} 38^{\prime} 17.72 " \mathrm{O}$ & $35^{\circ} 12^{\prime} 7.84^{\prime \prime} \mathrm{N}$ & $478 \mathrm{~m}$ \\
\hline Tichedrett & & Sidi Belabbes & $0^{\circ} 38^{\prime} 17.72 " \mathrm{O}$ & $35^{\circ} 12^{\prime} 7.84^{\prime \prime} \mathrm{N}$ & $478 \mathrm{~m}$ \\
\hline Faouara2 & & Sidi Belabbes & $0^{\circ} 38^{\prime} 17.72 " \mathrm{O}$ & $35^{\circ} 12^{\prime} 7.84^{\prime \prime} \mathrm{N}$ & $478 \mathrm{~m}$ \\
\hline Azrir & Adrar & Oueled Ali & $0^{\circ} 5^{\prime} 52.95^{\prime \prime O}$ & $28^{\circ} 42^{\prime} 51.56^{\prime \prime N}$ & $251 \mathrm{~m}$ \\
\hline Safra & & Oueled Ali & $0^{\circ} 5^{\prime} 52.95 " \mathrm{O}$ & $28^{\circ} 42^{\prime} 51.56^{\prime \prime} \mathrm{N}$ & $251 \mathrm{~m}$ \\
\hline Bourabaa & & Adrar & $0^{\circ} 11^{\prime} 12.99 " \mathrm{O}$ & $27^{\circ} 58^{\prime} 18.55^{\prime \prime} \mathrm{N}$ & $257 \mathrm{~m}$ \\
\hline Salt & & Oueled Ali & $0^{\circ} 5^{\prime} 52.95 " \mathrm{O}$ & $28^{\circ} 42^{\prime} 51.56 " \mathrm{~N}$ & $251 \mathrm{~m}$ \\
\hline Ras el mouch & & Oueled Ali & $0^{\circ} 5^{\prime} 52.95 " \mathrm{O}$ & $28^{\circ} 42^{\prime} 51.56 " \mathrm{~N}$ & $251 \mathrm{~m}$ \\
\hline Chater & & Kerzaz & $1^{\circ} 26^{\prime} 19.08^{\prime \prime O}$ & $29^{\circ} 27^{\prime} 44.71 " \mathrm{~N}$ & $387 \mathrm{~m}$ \\
\hline Bourabaa & & Kerzaz & $1^{\circ} 26^{\prime} 19.08^{\prime \prime O}$ & $29^{\circ} 27^{\prime} 44.71 " \mathrm{~N}$ & $387 \mathrm{~m}$ \\
\hline Chater 2 & & Adrar & $0^{\circ} 11^{\prime} 12.99 " \mathrm{O}$ & $27^{\circ} 58^{\prime} 18.55^{\prime \prime N}$ & $257 \mathrm{~m}$ \\
\hline Zraa beldi & & Zaouiet Konta & $0^{\circ} 12^{\prime} 3.68^{\prime \prime O}$ & $27^{\circ} 1334.73^{\prime \prime} \mathrm{N}$ & $204 \mathrm{~m}$ \\
\hline Ras el mouch & & Oueled Ali & $0^{\circ} 5^{\prime} 52.95 " \mathrm{O}$ & $28^{\circ} 42^{\prime} 51.56^{\prime \prime} \mathrm{N}$ & $251 \mathrm{~m}$ \\
\hline Ghir & Bechar & Bechar & $2^{\circ} 10^{\prime} 58.22^{\prime \prime O}$ & $31^{\circ} 33^{\prime} 28.58^{\prime \prime N}$ & $789 \mathrm{~m}$ \\
\hline Saida & & Bechar & $2^{\circ} 10^{\prime} 58.22^{\prime \prime O}$ & $31^{\circ} 3328.58^{\prime \prime N}$ & $789 m$ \\
\hline Safra3 & & Beni Abbes & $2^{\circ} 10^{\prime} 1.66^{\prime \prime O}$ & $30^{\circ} 7 ' 56.89^{\prime \prime} \mathrm{N}$ & $505 \mathrm{~m}$ \\
\hline Saida & & Beni Abbes & $2^{\circ} 10^{\prime} 1.66^{\prime \prime} \mathrm{O}$ & $30^{\circ} 7 ' 56.89^{\prime \prime} \mathrm{N}$ & $505 \mathrm{~m}$ \\
\hline Safra2 & & Beni Abbes & $2^{\circ} 10^{\prime} 1.66 " \mathrm{O}$ & $30^{\circ} 7{ }^{\prime} 56.89^{\prime \prime} \mathrm{N}$ & $505 \mathrm{~m}$ \\
\hline Zraa byad 1 & & Beni Abbes & $2^{\circ} 10^{\prime} 1.66^{\prime \prime O}$ & $30^{\circ} 7{ }^{\prime} 56.89^{\prime \prime} \mathrm{N}$ & $505 \mathrm{~m}$ \\
\hline Zraa byad 2 & & Bechar & $2^{\circ} 10^{\prime} 58.22 " \mathrm{O}$ & $31^{\circ} 3328.58^{\prime \prime N}$ & $789 \mathrm{~m}$ \\
\hline Rihane & Ain Defla & Khemiss Meliana & $2^{\circ} 12^{\prime} 49.78^{\prime \prime} \mathrm{E}$ & $36^{\circ} 15^{\prime} 12.70^{\prime \prime} \mathrm{N}$ & $281 \mathrm{~m}$ \\
\hline Saida & & Khemiss Meliana & $2^{\circ} 12^{\prime} 49.78^{\prime \prime} \mathrm{E}$ & $36^{\circ} 15^{\prime} 12.70^{\prime \prime} \mathrm{N}$ & $281 \mathrm{~m}$ \\
\hline Faouara & & Khemiss Meliana & $2^{\circ} 12^{\prime} 49.78^{\prime \prime} \mathrm{E}$ & $36^{\circ} 15^{\prime} 12.70^{\prime \prime} \mathrm{N}$ & $281 \mathrm{~m}$ \\
\hline Saida & Media & Beni Slimane & $2^{\circ} 56^{\prime} 15.27 " \mathrm{E}$ & $36^{\circ} 3^{\prime} 12.55^{\prime \prime} \mathrm{N}$ & $588 \mathrm{~m}$ \\
\hline Rihane & & Beni Slimane & $2^{\circ} 56^{\prime} 15.27 " \mathrm{E}$ & $36^{\circ} 3^{\prime} 12.55^{\prime \prime} \mathrm{N}$ & $588 \mathrm{~m}$ \\
\hline Tichedrett & & Beni Slimane & $2^{\circ} 56^{\prime} 15.27 " \mathrm{E}$ & $36^{\circ} 3^{\prime} 12.55^{\prime \prime} \mathrm{N}$ & $588 \mathrm{~m}$ \\
\hline
\end{tabular}

The relative diversity index $\left(\mathrm{H}^{\prime}\right)$ reaches its minimum value, which is zero for monomorphic characters. Moreover, the value of this index increases with the degree of polymorphism and reaches a maximum value (1) when all the phenotypic classes are present in equal frequencies. Using R. Software a multivariate analysis was performed to discriminate accessions with Hierarchical Ascending Classification (HAC) and principal component analysis. Hierarchical Ascendant Classification, using the Facto MineR software (version R-2.15.3) it is used to better classification of the 33 accessions of barleyPrincipal component analysis was carried out on the correlation matrix with Facto MineR software (version R-2.15.3).

\section{RESULTS AND DISCUSSION}

\section{Relative diversity index of the qualitative and quantitative characters}

The relative diversity index $\left(\mathrm{H}^{\prime}\right.$ average) of all studied accessions is 0.74 for the quantitative characters and 0.53 for the qualitative characters (Table 2 and 3). At population level, for the quantitative characters, this index ranges from0.51 to 0.94 respectively for the accessions Saida and Bourabaa. This index ranges from 0.52 of stem length and 0.85 for the width of the spike (Table 2). For the qualitative characters, this index ranges from 0.39 to 0.65 respectively for the accessions (Ascad, 2 Rang) and Saida. This index ranges from 0.0 of number of rows and 0.74 for longer compared to ear (Table 3 ).

According to all the features and all accessions: the highest diversity index $\left(\mathrm{H}^{\prime}=0.99\right)$ was obtained for grain per ear density character of the Ascad variety at Sidi Belabbes region and the Ghir variety at Bechar.Among the qualitative characters, the same value was found for the character Grain: hairiness of ventral furrow of the Zraa Beldi accession at Adrar region.

\section{Relative index diversity of different characters depending on the region}

The index of Shannon and Weaver was calculated from the different characters in six regions surveyed: Tlemcen, 
Sidi Belabbes, Ain Defla, Media, Bechar and Adrar (Table 2). For quantitative characters concerning overall, the region of Adrar had the highest average diversity index with 0.81 , followed by Sidi Belabbes region (0.80). Lowest average value was found in Tlemcen (0.54). For qualitative characters, Media experienced the highest average diversity index with 0.57 followed by the Bechar region with 0.54 . The lowest average value was found at Sidi Belabbes region (0.50) (Table 3). For qualitative characters, the highest diversity indices $\left(H^{\prime} \geq 0,60\right)$ are obtained from six accessions, intermediate values $(0,40 \leq \mathrm{H}<0.60)$ were obtained from twenty-six accessions, and the lower values $(0,10 \leq \mathrm{H}<0.40)$ were observed from one accession. The average $H^{\prime}$ between regions ranged from 0.35 in Median spikelet to 0.74 in Awn: length compared to ear.

To analyze the structure of the genetic diversity among the set of accessions that constitutes this collection, principal component analysis was performed on the matrix of accessions by characters, separately for quantitative and qualitative traits. The PCA of barley accessions for the quantitative traits (Figure 2) explained $65.50 \%$ of the variation.

The second principal component calculated for the qualitative characters (figure03) represented $41.2 \%$ of the variation.

Table 2. Estimate of Shannon diversity indices for quantitative characteristics of Barley accessions, in Algeria

\begin{tabular}{|c|c|c|c|c|c|c|c|c|c|c|c|c|c|}
\hline Variety & PL & SL & EL & EL/excA & $\mathbf{A L}$ & $\mathbf{E W}$ & DE & NGE & DG/E & GW & $\mathbf{L G}$ & $\mathbf{R} / \mathbf{l s}$ & $H^{\prime}$ mean \\
\hline \multicolumn{14}{|l|}{ Sidi Belabbes } \\
\hline Ascad Sba & 0.86 & 0.93 & 0.87 & 0.95 & 0.85 & 0.86 & 0.87 & 0.91 & 0.99 & 0.38 & 0.91 & 0.85 & 0.85 \\
\hline Tichedrett Sba & 0.76 & 0.79 & 0.80 & 0.82 & 0.81 & 0.96 & 0.94 & 0.96 & 0.96 & 0.89 & 0.91 & 0.95 & 0.88 \\
\hline 2 Rangs Sba & 0.42 & 0.44 & 0.48 & 0.77 & 0.44 & 0.78 & 0.76 & 0.62 & 0.72 & 0.31 & 0.89 & 0.98 & 0.63 \\
\hline Saida Sba & 0.67 & 0.61 & 0.77 & 0.93 & 0.71 & 0.96 & 0.67 & 0.73 & 0.65 & 0.91 & 0.75 & 0.58 & 0.75 \\
\hline Saida S.Lahsen & 0.74 & 0.67 & 0.97 & 0.82 & 0.88 & 0.64 & 0.88 & 0.98 & 0.90 & 0.71 & 0.76 & 0.89 & 0.82 \\
\hline Rihane Sba & 0.68 & 0.76 & 0.71 & 0.53 & 0.90 & 0.90 & 0.93 & 0.97 & 0.92 & 0.70 & 0.98 & 0.52 & 0.79 \\
\hline Fawara Sba & 0.84 & 0.76 & 0.98 & 0.81 & 0.91 & 0.91 & 0.98 & 0.78 & 0.99 & 0.84 & 0.79 & 0.91 & 0.88 \\
\hline $\begin{array}{l}\text { Mean Sidi Belabbes Region } \\
\text { Tlemcen }\end{array}$ & 0.71 & 0.71 & 0.80 & 0.80 & 0.78 & 0.86 & 0.85 & 0.85 & 0.87 & 0.68 & 0.86 & 0.81 & 0.80 \\
\hline Saida G3 & 0.50 & 0.71 & 0.32 & 0.49 & 0.66 & 0.68 & 0.28 & 0.70 & 0.28 & 0.28 & 0.68 & 0.91 & 0.54 \\
\hline Saida G4 & 0.58 & 0.00 & 0.94 & 0.98 & 0.87 & 0.42 & 0.38 & 0.61 & 0.49 & 0.92 & 0.34 & 0.38 & 0.58 \\
\hline Saida S. Abdli & 0.39 & 0.58 & 0.48 & 0.77 & 0.44 & 0.80 & 0.49 & 0.60 & 0.72 & 0.44 & 0.18 & 0.18 & 0.51 \\
\hline Mean Tlemcen Region & 0.49 & 0.43 & 0.58 & 0.75 & 0.66 & 0.63 & 0.38 & 0.64 & 0.50 & 0.55 & 0.40 & 0.49 & 0.54 \\
\hline \multicolumn{14}{|l|}{ Adrar } \\
\hline Azrir Ad & 0.63 & 0.63 & 0.78 & 0.90 & 0.91 & 0.85 & 0.55 & 0.61 & 0.65 & 0.97 & 0.93 & 0.91 & 0.78 \\
\hline Ras Elmouch Ad & 0.11 & 0.11 & 0.89 & 0.95 & 0.93 & 0.84 & 0.92 & 0.95 & 0.90 & 0.91 & 0.80 & 0.93 & 0.77 \\
\hline Ras Elmouch O.Ali & 0.28 & 0.00 & 0.87 & 0.93 & 0.80 & 0.85 & 0.78 & 0.57 & 0.70 & 0.91 & 0.95 & 0.78 & 0.70 \\
\hline Saltt Ad & 0.71 & 0.70 & 0.64 & 0.74 & 0.77 & 0.91 & 0.89 & 0.90 & 0.82 & 0.72 & 0.82 & 0.91 & 0.79 \\
\hline Safra O.Ali & 0.78 & 0.78 & 1.00 & 0.59 & 0.91 & 1.00 & 0.87 & 0.99 & 0.85 & 0.92 & 0.94 & 0.92 & 0.88 \\
\hline Bourabaa Ad & 0.98 & 0.98 & 0.93 & 0.93 & 0.79 & 0.93 & 0.82 & 0.99 & 0.80 & 0.90 & 0.49 & 0.90 & 0.87 \\
\hline Bourabaa Ker & 0.98 & 0.95 & 0.97 & 0.82 & 0.97 & 0.91 & 0.97 & 0.92 & 0.93 & 0.94 & 0.88 & 0.98 & 0.94 \\
\hline Zraa Beldi Ad & 0.58 & 0.54 & 0.98 & 0.97 & 0.76 & 0.32 & 0.71 & 0.72 & 0.58 & 0.78 & 0.77 & 0.96 & 0.72 \\
\hline Chater Ad & 0.76 & 0.68 & 0.78 & 0.68 & 0.45 & 0.99 & 0.89 & 0.95 & 0.94 & 0.96 & 0.77 & 0.86 & 0.81 \\
\hline Chater Ker & 0.66 & 0.63 & 0.77 & 0.88 & 0.67 & 0.82 & 0.98 & 0.97 & 0.95 & 0.88 & 0.87 & 0.88 & 0.83 \\
\hline Mean Adrar Region & 0.65 & 0.60 & 0.86 & 0.84 & 0.80 & 0.84 & 0.84 & 0.86 & 0.81 & 0.89 & 0.82 & 0.90 & 0.81 \\
\hline \multicolumn{14}{|l|}{ Bechar } \\
\hline Zraa Byad Bechar & 0.11 & 0.00 & 0.76 & 0.99 & 0.60 & 0.91 & 0.95 & 0.89 & 0.86 & 0.92 & 0.67 & 0.58 & 0.69 \\
\hline Zraabyad B.Abbes & 0.18 & 0.18 & 0.48 & 0.54 & 0.59 & 0.97 & 0.60 & 0.70 & 0.69 & 0.81 & 0.97 & 0.68 & 0.62 \\
\hline Saida Bechar & 0.36 & 0.42 & 0.81 & 0.84 & 0.82 & 0.86 & 0.77 & 0.85 & 0.84 & 0.80 & 0.83 & 0.94 & 0.76 \\
\hline Saida B.Abbes & 0.11 & 0.52 & 0.93 & 0.83 & 0.94 & 0.75 & 0.16 & 0.85 & 0.78 & 0.94 & 0.78 & 0.00 & 0.63 \\
\hline Ghir Bechar & 0.97 & 0.95 & 0.89 & 0.84 & 0.72 & 0.86 & 0.98 & 0.98 & 0.99 & 0.89 & 0.80 & 0.91 & 0.90 \\
\hline Safra Bechar & 0.11 & 0.11 & 0.68 & 0.58 & 0.66 & 0.87 & 0.83 & 0.71 & 0.91 & 0.88 & 0.87 & 0.65 & 0.66 \\
\hline Safra B.Abbes & 0.49 & 0.46 & 0.98 & 0.74 & 0.75 & 0.84 & 0.82 & 0.61 & 0.79 & 0.82 & 0.63 & 0.94 & 0.74 \\
\hline Mean Bechar Region & 0.33 & 0.38 & 0.79 & 0.77 & 0.73 & 0.86 & 0.73 & 0.80 & 0.84 & 0.87 & 0.79 & 0.67 & 0.71 \\
\hline \multicolumn{14}{|l|}{ Ain Defla } \\
\hline Rihane Ain.D & 0.68 & 0.71 & 0.74 & 0.58 & 0.80 & 0.94 & 0.91 & 0.66 & 0.94 & 0.97 & 0.86 & 0.11 & 0.74 \\
\hline Fawara Ain.D & 0.11 & 0.00 & 0.76 & 0.59 & 0.68 & 0.97 & 0.85 & 0.98 & 0.96 & 0.97 & 0.97 & 0.56 & 0.70 \\
\hline Saida Ain.D & 0.49 & 0.65 & 0.76 & 0.75 & 0.84 & 0.89 & 0.93 & 0.78 & 0.96 & 0.92 & 0.91 & 0.77 & 0.81 \\
\hline Mean Ain Defla Region & 0.43 & 0.46 & 0.75 & 0.64 & 0.77 & 0.93 & 0.90 & 0.81 & 0.96 & 0.95 & 0.91 & 0.48 & 0.75 \\
\hline \multicolumn{14}{|l|}{ Media } \\
\hline Saida M & 0.91 & 0.82 & 0.98 & 0.93 & 0.97 & 0.91 & 0.90 & 0.95 & 0.93 & 0.98 & 0.86 & 0.89 & 0.92 \\
\hline Rihane M & 0.42 & 0.42 & 0.58 & 0.64 & 0.79 & 0.95 & 0.84 & 0.44 & 0.82 & 0.77 & 0.71 & 0.54 & 0.66 \\
\hline Tichedrett M & 0.18 & 0.00 & 0.84 & 0.78 & 0.67 & 0.98 & 0.57 & 0.36 & 0.68 & 0.95 & 0.28 & 0.52 & 0.57 \\
\hline MeanAin Defla Region & 0.50 & 0.41 & 0.80 & 0.78 & 0.81 & 0.95 & 0.77 & 0.58 & 0.81 & 0.90 & 0.61 & 0.65 & 0.71 \\
\hline Mean & 0.54 & 0.52 & 0.79 & 0.78 & 0.76 & 0.85 & 0.77 & 0.79 & 0.81 & 0.81 & 0.77 & 0.72 & 0.74 \\
\hline
\end{tabular}


Note: PL: Plant length,SL: stem length,EL: ear length, EL/excA: ear length (excluding awns), NG/E: number of grains per ear, AL:, awn length, DG/E: density of grains per ear, EW: ear width, ED: ear density, GW: grain width (mm), GL: grain length (mm), R/ls: rachis: length of first segment $(\mathrm{mm})$. 
BIODIVERSITAS

ISSN: 1412-033X

Volume 20, Number 12, December 2019

-ISSN: 2085-4722

Pages: 3794-3803

DOI: $10.13057 /$ biodiv/d201244

Table 3. Estimate of Shannon diversity indices for qualitative characteristics of Barley accessions in Algeria

\begin{tabular}{|c|c|c|c|c|c|c|c|c|c|c|c|c|c|c|c|c|c|c|c|}
\hline Variétas & EA & $\mathbf{E S}$ & HVF & CFS & ATE & MS/G & SL & D L & RH & SB & ing & $\overline{\mathbf{B A}}$ & Pig & $\overline{A C}$ & $\overline{\mathrm{KC}}$ & AlE & ESE & NR & H'Mean \\
\hline Sidi Belabbes & & & & & & & & & & & & & & & & & & & \\
\hline $\begin{array}{l}\text { Ascad Sba } \\
\text { Tichedrett Sha }\end{array}$ & 0.54 & $\begin{array}{l}0.22 \\
0.87\end{array}$ & 0.35 & $\begin{array}{l}0.80 \\
0.88\end{array}$ & 0.56 & 0.61 & 0.20 & 0.57 & $\begin{array}{l}0.21 \\
0.79\end{array}$ & 0.35 & 0.53 & 0.21 & 0.35 & 0.22 & 0.30 & 0.58 & 0.69 & 0.00 & 0.39 \\
\hline $\begin{array}{l}\text { Tichedrett Sba } \\
2 \text { Rang Sba }\end{array}$ & $\begin{array}{l}0.40 \\
0.65\end{array}$ & $\begin{array}{l}0.81 \\
0.30\end{array}$ & $\begin{array}{l}0.35 \\
0.21\end{array}$ & $\begin{array}{l}0.88 \\
0.36\end{array}$ & $\begin{array}{l}0.73 \\
0.41\end{array}$ & $\begin{array}{l}0.72 \\
0.45\end{array}$ & $\begin{array}{l}0.66 \\
0.09\end{array}$ & $\begin{array}{l}0.21 \\
0.65\end{array}$ & $\begin{array}{l}0.79 \\
0.21\end{array}$ & $\begin{array}{l}0.84 \\
0.35\end{array}$ & $\begin{array}{l}0.45 \\
0.78\end{array}$ & 0.98 & 0.51 & 0.61 & 0.86 & 0.82 & $\begin{array}{l}0.49 \\
0.41\end{array}$ & 0.00 & $\begin{array}{l}0.62 \\
0.39\end{array}$ \\
\hline $\begin{array}{l}\text { 2 Kang soa } \\
\text { Saida Sba }\end{array}$ & 0.82 & 0.58 & 0.73 & 0.89 & 0.36 & 0.62 & 0.46 & 0.65 & 0.00 & 0.00 & 0.56 & 0.21 & 0.58 & 0.61 & 0.57 & $\begin{array}{l}0.00 \\
1.00\end{array}$ & 0.36 & 0.00 & 0.50 \\
\hline Saida S.Lahcen & 0.42 & 0.61 & 0.35 & 0.80 & 0.81 & 0.80 & 0.51 & 0.92 & 0.95 & 0.92 & 0.51 & 0.98 & 0.92 & 0.80 & 0.13 & 0.58 & 0.61 & 0.00 & 0.65 \\
\hline Rihane Sba & 0.42 & 0.58 & 0.47 & 0.45 & 0.78 & 0.53 & 0.15 & 0.57 & 0.79 & 0.79 & 0.13 & 0.35 & 0.51 & 0.22 & 0.22 & 0.73 & 0.73 & 0.00 & 0.47 \\
\hline Faouara Sba & 0.56 & 0.41 & 0.73 & 0.32 & 0.45 & 0.85 & 0.20 & 0.35 & 0.73 & 0.73 & 0.00 & 0.73 & 0.00 & 0.58 & 0.51 & 0.61 & 0.73 & 0.00 & 0.47 \\
\hline Avr Sidi-B Region & 0.56 & 0.51 & 0.46 & 0.64 & 0.59 & 0.65 & 0.32 & 0.56 & 0.52 & 0.57 & 0.42 & 0.63 & 0.45 & 0.44 & 0.37 & 0.73 & 0.57 & 0.00 & 0.49 \\
\hline $\begin{array}{l}\text { Tlemcen } \\
\text { Saida G3 }\end{array}$ & 0.54 & 0.60 & 0.0 & 0.68 & 0 & 51 & 0.33 & 0.73 & 0.73 & 0.65 & 0.58 & 0.35 & 0.31 & 22 & 0.74 & 0.81 & 0.30 & 0.00 & 0.49 \\
\hline Saida G4 & 0.69 & 0.36 & 0.00 & 0.62 & 0.88 & 0.62 & 0.24 & 0.35 & 0.65 & 0.35 & 0.63 & 0.35 & 0.40 & 0.81 & 0.22 & $\begin{array}{l}0.01 \\
0.73\end{array}$ & 0.60 & 0.00 & 0.47 \\
\hline Saida S. Abdli & 0.62 & 0.45 & 0.95 & 0.79 & 0.74 & 0.70 & 0.24 & 0.84 & 0.98 & 0.65 & 0.68 & 0.00 & 0.97 & 0.62 & 0.74 & 0.80 & 0.30 & 0.00 & 0.62 \\
\hline Meantlemcen Region & 0.62 & 0.47 & 0.32 & 0.70 & 0.78 & 0.64 & 0.27 & 0.64 & 0.78 & 0.55 & 0.63 & 0.24 & 0.56 & 0.55 & 0.57 & 0.78 & 0.40 & 0.00 & 0.53 \\
\hline $\begin{array}{l}\text { Adrar } \\
\text { Azrir Ad }\end{array}$ & 0.36 & 0.62 & 0.35 & 0.60 & 063 & 045 & 052 & 035 & 070 & 073 & 063 & 000 & 001 & 000 & 0.63 & 007 & 073 & 000 & 0.51 \\
\hline Rassel Mouch Ad & 0.55 & 0.41 & 0.00 & 0.30 & 0.96 & 0.74 & 0.51 & 0.78 & 0.78 & 0.57 & 0.43 & 0.97 & 0.56 & 0.13 & 0.61 & 0.94 & 0.56 & 0.00 & 0.54 \\
\hline Rass El Mouch O.Ali & 0.40 & 0.56 & 0.65 & 0.30 & 0.74 & 0.61 & 0.33 & 0.47 & 0.35 & 0.21 & 0.70 & 0.00 & 0.57 & 0.70 & 0.74 & 0.85 & 0.73 & 0.00 & 0.50 \\
\hline Sallt Ad & 0.42 & 0.60 & 0.33 & 0.85 & 0.22 & 0.22 & 0.29 & 0.65 & 0.21 & 0.35 & 0.72 & 0.95 & 0.85 & 0.30 & 0.36 & 0.73 & 0.73 & 0.00 & 0.49 \\
\hline Safra O.Ali & 0.43 & 0.62 & 0.00 & 0.62 & 0.73 & 0.62 & 0.33 & 0.57 & 0.73 & 0.21 & 0.45 & 0.98 & 0.45 & 0.00 & 0.62 & 0.60 & 0.45 & 0.00 & 0.47 \\
\hline Bour & 0.43 & 0.61 & 0.00 & 0.81 & 0.74 & 0.61 & 0.44 & 0.79 & 0.47 & 0.35 & 0.68 & 0.21 & 0.58 & 0.61 & 0.30 & 0.85 & 0.76 & 0.00 & 0.51 \\
\hline a Ker & 0.40 & 0.36 & 0.39 & 0.57 & 0.92 & 0.60 & 0.54 & 0.57 & 0.47 & 0.57 & 0.81 & 0.73 & 0.00 & 0.45 & 0.51 & 0.81 & 0.36 & 0.00 & 0.50 \\
\hline di Ad & 0.52 & 0.85 & 0.99 & 0.56 & 0.92 & 0.94 & 0.24 & 0.72 & 0.47 & 0.35 & 0.86 & 0.97 & 0.38 & 0.36 & 0.35 & 0.63 & 0.73 & 0.00 & 0.60 \\
\hline Cha & 43 & 0.49 & 0 & 0. & 0.80 & 0. & 0.15 & 0.36 & 0.21 & 0 & 0. & 0.21 & 0.85 & 0.30 & 0.22 & 0.60 & 0.41 & 0.00 & 0.43 \\
\hline Cha & .43 & 0.88 & 0.57 & 0.81 & 0.92 & 0.74 & 0.09 & 0.88 & 0.21 & 0.47 & 0.43 & 0.97 & 0.98 & 0.63 & 0.63 & 0.87 & 0.70 & 0.00 & 0.62 \\
\hline $\begin{array}{l}\text { Mean Adrar Region } \\
\text { Bechar }\end{array}$ & 0.44 & 0.60 & 0.34 & 0.62 & $0.7 \overline{6}$ & 0.65 & 0.34 & 0.61 & 0.47 & 0.40 & 0.63 & 0.60 & 0.61 & 0.35 & 0.50 & 0.78 & 0.62 & 0.00 & 0.52 \\
\hline yad Becha & 40 & 0.63 & 0.3 & 0.30 & 0.6 & 0.84 & 0.20 & 0 & 0.65 & 0.57 & 0.8 & 0.73 & 0.00 & 0.41 & 0.51 & 0.83 & 0.76 & 0.00 & 0.54 \\
\hline $\mathrm{ad}$ B.Ab & 0 & 0.58 & 0 & 0.90 & 0. & 0. & 0.61 & 0. & 0.73 & 0.73 & 0.70 & 0.79 & 0.98 & 0.30 & 0.81 & 0 & 0.68 & 0.00 & 0.63 \\
\hline Said & 0 & 0.60 & 021 & 0.30 & 0 & & 0.66 & 0 & 0.98 & 0.79 & 0.00 & 0.73 & 0.00 & 0.41 & 0.54 & & 0.73 & 0.00 & 0.50 \\
\hline Sai & & 0. & & 0. & 0. & & 0. & 0. & 0.92 & 0. & 0. & 0.47 & 0. & 0.81 & & & & & 0.59 \\
\hline Ghir & 3 & 0.62 & 0.6 & 0.90 & 0.7 & 0. & 0. & 0. & 0.35 & 0.35 & 0. & 0.21 & 0. & 0. & 0. & & 0.36 & 0.00 & 0.52 \\
\hline & 2 & 0.60 & 0 & 0.63 & 0. & & 0. & 0. & 0.79 & 0 & 0. & 0.92 & 0 & & 0 & & 0.41 & 0.00 & 0.59 \\
\hline Safr & & 0.53 & 0.0 & 0.30 & 0.7 & 0.49 & 0.20 & 0. & 0.35 & 0.47 & 0.30 & $0.5 \overline{3}$ & 0.59 & 0.45 & 0.81 & 0.72 & 0.70 & 0.00 & 0.43 \\
\hline har Region & 0.39 & 0.59 & 0.30 & 0.58 & 0.72 & 0.68 & 0.47 & 0.65 & 0.68 & 0.65 & 0.48 & 0.63 & 0.57 & 0.45 & 0.58 & 0.71 & 0.62 & 0.00 & 0.54 \\
\hline $\begin{array}{l}\text { Ain } \\
\text { Riha }\end{array}$ & & 0.63 & 0 & 0.79 & & & 0.33 & & 0. & 0 & 0 & 0. & 0.57 & 0. & 0.73 & 0.70 & 0.76 & 0.00 & 0.59 \\
\hline n.D & 0.63 & 0.53 & & 0.66 & 0.9 & 0. & 0.13 & 0. & 0.79 & 0.57 & 0. & 0.2 & 0.00 & 0. & 0.30 & & 0.53 & 0.00 & 0.52 \\
\hline Said & 0. & 0.41 & 0.8 & 0.3 & 0.6 & & 0.15 & 0. & 0.65 & & 0. & 0.47 & 0.71 & 0.62 & 0.26 & & 0.76 & 0.00 & 0.52 \\
\hline Mean Ain Defla region & $0.4 \mathrm{C}$ & 0.52 & 0.78 & 0.58 & 0.77 & 0.73 & 0.20 & 0.74 & 0.64 & 0.53 & 0.48 & 0.55 & 0.43 & 0.55 & 0.43 & 0.68 & 0.68 & 0.00 & 0.54 \\
\hline $\begin{array}{l}\text { Med } \\
\text { Said }\end{array}$ & ( & & & & & & & & & & 0 & & & & 0 & & 0. & U & 0.52 \\
\hline & 0.41 & 0.70 & & & & 0. & 0. & & 0.92 & 0. & 0. & 0.65 & 0.71 & 0. & 0 & & 0.58 & 0.00 & 0.59 \\
\hline rett $M$ & 0.42 & 0.56 & 0.2 & 0.92 & 0. & 0. & 0.21 & 0. & 0.72 & 0.84 & 0. & 0.97 & 0.54 & 0.81 & 0.51 & 0.97 & 0.60 & 0.00 & 0.62 \\
\hline f Media re & 0.42 & 0.62 & 0.3 & 0.90 & 0 . & 0. & 0.27 & 0. & 0.67 & 0.5 & 0. & 0.80 & 0.59 & 0.57 & 0.42 & & 0.47 & 0.00 & 0.57 \\
\hline Mean & 0.47 & 0.56 & 0.41 & 0.64 & 0.73 & 0.67 & 0.35 & 0.63 & 0.59 & 0.53 & 0.53 & 0.58 & 0.53 & 0.44 & 0.48 & 0.74 & 0.58 & 0.00 & 0.53 \\
\hline
\end{tabular}

WFS: curvature of first segment), ATE: sterile spikelet attitude(in mid-third of ear), MS/G: media spikelet lenght of glume and its awn relative to grain, SL: spiculation of inner lateral nerves of lemma, DL: disposition of lodicules, RH: Grain: rachilla hair type, SB: Lemma: shape of base,

zig: alignment of articles at the middle tier of ear, BA: bosse of articles at the middle tier of ear, pig: Awns: anthocyanin coloration of tips, AC: Awn color, CK: kennel: color of aleurone layer, AIE: Awn: length compared to ear, ESE: ear: shape of the extremity, NR: number of rows. 


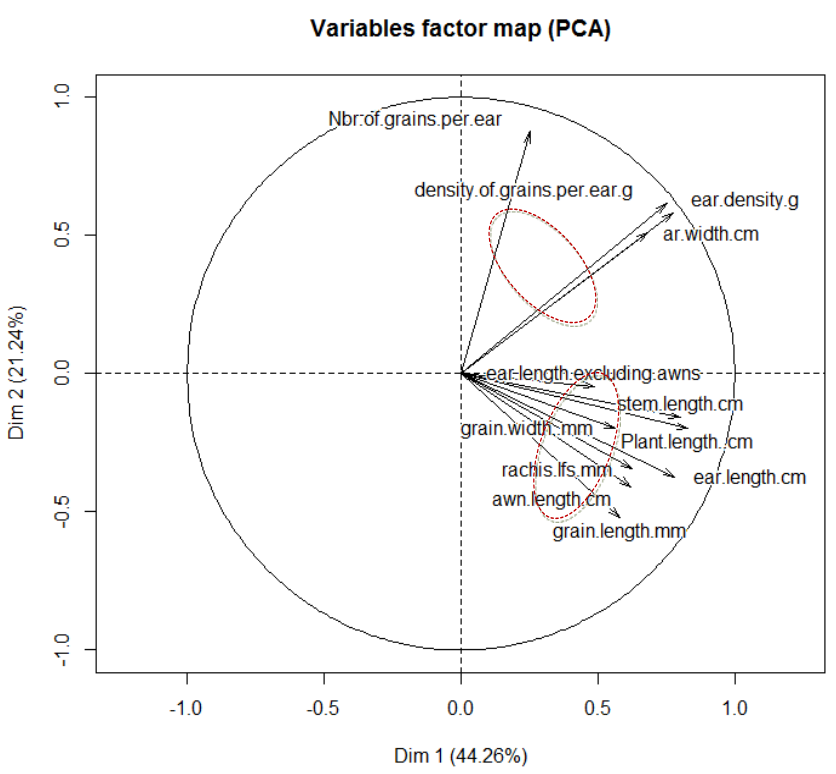

Figure. 2. PCA of different quantitative characters of Barley in Algeria

\section{Genetic relationship among the Algerian Barley landraces}

The dendrogram describes the relationship among the landraces based on the quantitative traits (Figure 4), dividing the accessions into two main groups, the first group comprised a first subgroup shown the mixture of barley accessions from Adrar and Bechar. Within the second subgroup contained only accessions from Adrar excepet for 2 Rangs accession which is from Sidi Belabbes. The second group could be subdivided on 2 subgroup where the first one is formed by accession namely Saida from the regions of Tlemcen, Sidi Belabbes, Media, Ain Defla and Bechar. Whereas the second subgroup includes the mixture of barley accessions from Sidi Belabbes, Media and Ain Defla.

For the qualitative characters (Figure 5), the dendrogram is divided into two major groups, the first one gathered the first subgroup with accessions from Adrar in the first class and the accessions of Bechar, Sidi Belabbes, and Media in the second class. Whereas the second subgroup gathered the accessions from Tlemcen in the first class, the accessions from Sidi Belabbes (except Saida accession from Ain Defla) and accession from Ain Defla in the second. The second group could be divided also into 2 subgroups where the first one contains Rihana accessions from Sidi Belabbes, Media accession, and Ain Defla accession. Where the second subgroup is formed by Tichedrett accessions from Sid Belabbes and Media accession in the first class and mixture of barley accessions

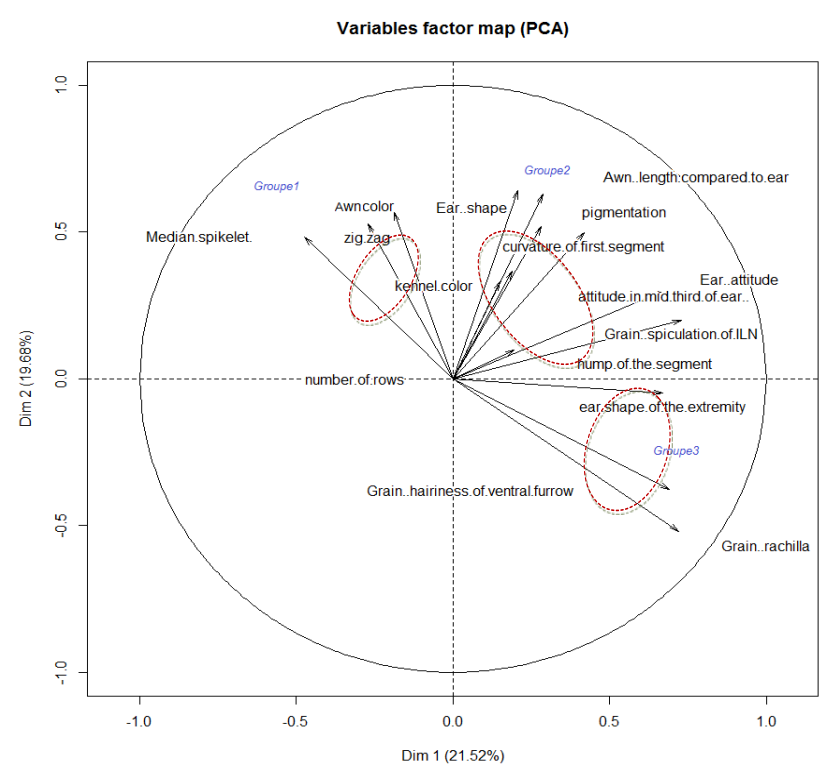

Figure. 3. PCA of different qualitative characters of Barley in Algeria

from Adrar, Sidi Belabbes and Ain Defla in the second class.

\section{Discussion}

In general, barley accessions studied had a high diversity index $\left(\mathrm{H}^{\prime}\right)$ with lower values in qualitative than in quantitative characters. Overall accessions, the $\mathrm{H}^{\prime}$ average was 0.74 for quantitative characters, this diversity is close to that reported in several studies (Dejene et al.2010, Jaradat et al.2004 and Mekonnon et al.2015). Analyses of diversity pattern, among accessions from different regions for quantitative characters, revealed existence of morphological diversity within regions due to differences in agro-ecological conditions (Mekonnon et al.2015). Concerning, the qualitative characters, the index $\mathrm{H}^{\prime}$ average (0.53) was lower than those reported by Derbew et al. (2013) for Ethiopian barley where $\mathrm{H}^{\prime}$ value was 0.63 and by Gegnaw and Hadado (2014) where H' value was 0.59 .

At population level, the highest $\mathrm{H}^{\prime}$ obtained from Bourabaa accession (Adrar region) with (0.94) and lowest from Saida accession (Tlemcen region) with $(0.51)$ for the quantitative characters. Several factors could be involved in explaining the difference in Shannon diversity indices values between localities, including natural factors such as adaptation to local conditions and human factors mainly farmers' preference (Belhadj et al. 2015). Agro-climatic features of the site (altitude, rainfall, temperature) had a significant and positive impact on diversity and area allocation of barley (Fetien et al. 2009). For qualitative characters, the index H'ranged from 0.39 for Ascad and 2 
Rangs accessions in the Sidi Belabbes region to 0.65 for Saida accession in the same region.

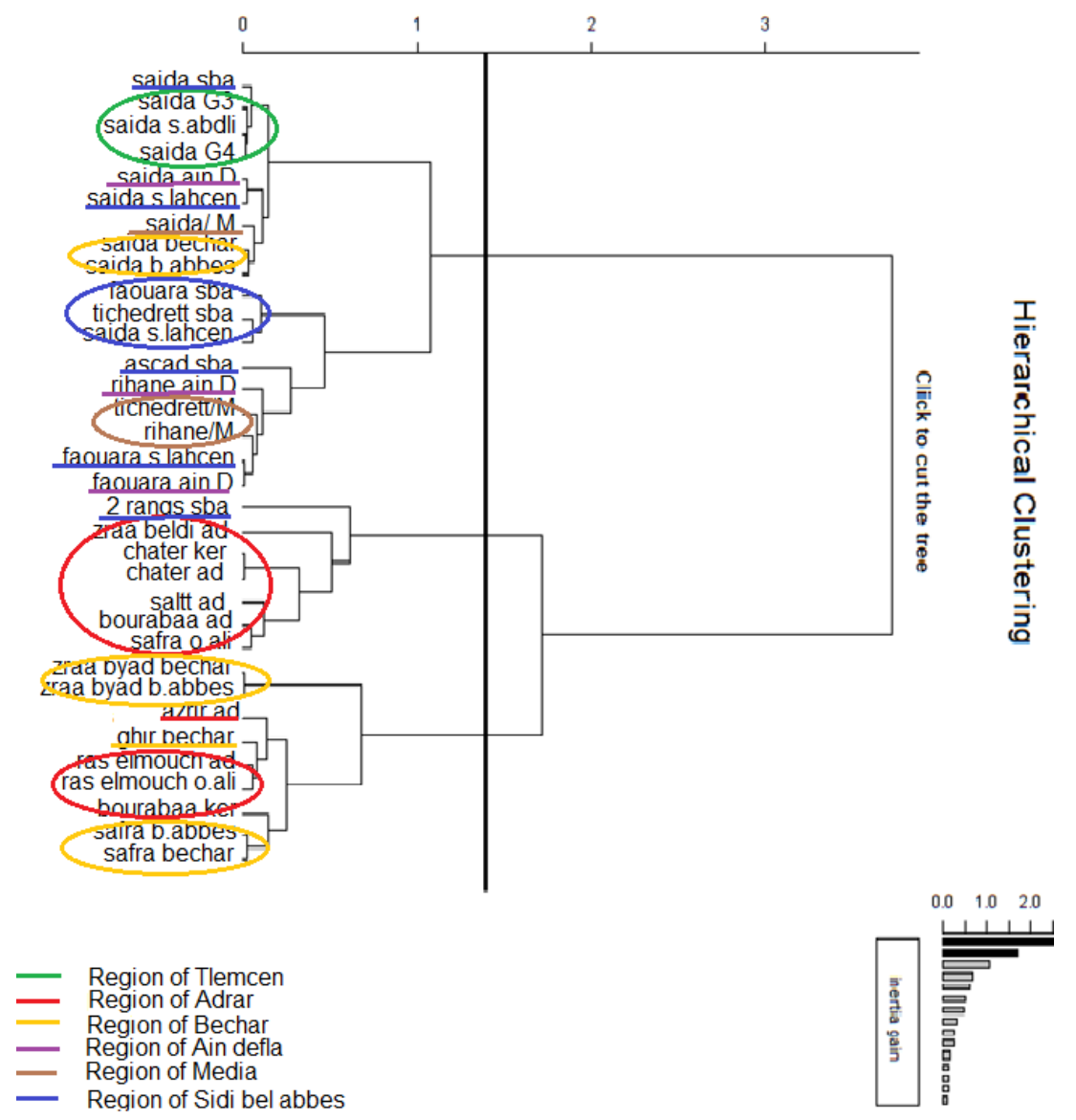

Figure. 4. Hierarchical Ascendant Classification (HAC) for quantitative characters of Barley in Algeria

The lowest diversity index of the quantitative characteristics was observed for stem length (0.52) and the highest is in ear width (0.85). The degree of variation for characters differed with districts and altitudes from where the landraces populations originated (Gegnaw and Hadado 2014). The indices of density of grains per ear and grain width are equal with $\left(\mathrm{H}^{\prime}=0.81\right)$. Concerning the density of ear, we have found $\left(\mathrm{H}^{\prime}=0.77\right)$ which is near to the one reported by Cross.1994 $\left(\mathrm{H}^{\prime}=0.78\right)$, with 20 entries from Morocco, Portugal, and Spain, from the New Zealand but lower than obtained by Tolbert et al.(1979) $\left(\mathrm{H}^{\prime}=0.92\right)$, with 63 Spanish landraces. For the length of plant, we found an index $\left(\mathrm{H}^{\prime}=0,54\right)$ which was lower than the one found by Fetien et al (2009) of a barley population in the north of Ethiopia with an index of 0.63 . For ear length (excluding awns), ear length and density of grains per ear, we found $\left(H^{\prime}=0.78\right),\left(H^{\prime}=0.79\right)$ and $\left(H^{\prime}=0.81\right)$ respectively which are higher than those found by Bellatreche et al. (2017) with $\left(\mathrm{H}^{\prime}=0.59\right), \quad\left(\mathrm{H}^{\prime}=0.46\right)$ and $\left(\mathrm{H}^{\prime}=0.58\right)$ respectively, for Algerian accessions of bread wheat. As far as the three characteristics are concerned: rachis: stem length $\left(H^{\prime}=0.52\right)$, length of first segment $\left(H^{\prime}=0.72\right)$, grain width $\left(\mathrm{H}^{\prime}=0.81\right)$ and grain length $\left(\mathrm{H}^{\prime}=0.77\right)$ and after consulting several databases we have not found similar work.

Papa et al. (1998) also found geography- dependent distribution of growth habit for local barleys in the Mediterranean region (Sardinia), though the adaptive explanation was not evident in their case.

For qualitative characters, the number of rows we found $\left(\mathrm{H}^{\prime}=0.00\right)$ which was different than reported by Cross (1994) with $\left(\mathrm{H}^{\prime}=0.36\right)$ and by Derbew et al. (2013) with $\left(\mathrm{H}^{\prime}=0.75\right)$. Concerning the kernel color, we found a value $\left(H^{\prime}=0.48\right)$ which was lower than found by Derbew et al.(2013) with $\left(\mathrm{H}^{\prime}=0.64\right)$, Fetien et al .(2009) with $\left(\mathrm{H}^{\prime}=\right.$ $0.85)$, and by Tolbert et al. (1979) with 40 entries from Algeria, Tunisia, Morocco, and Egypt with $\left(\mathrm{H}^{\prime}=0.71\right)$, an apparent exists association between phenotype and utility, especially kernel color and human consumption (Asfaw 1988). Human/conscious selection for white against the pigmented strains may be one of the factors responsible for the striking preponderance of the white phenotypic class( Demissie and Bjqrnstad,1996). For Rachilla hairs $\left(\mathrm{H}^{\prime}=\right.$ 0.59) value lower than ours was found by Tolbertet al. $1979\left(\mathrm{H}^{\prime}=0.88\right)$ and by Negassa et al.1985 $\left(\mathrm{H}^{\prime}=0.92\right)$, and by Cross 1994 . $\left(H^{\prime}=1.00\right)$. The rachilla hair trait has been 
considered as selectively neutral, with no adaptive significance (Takahash 1995).

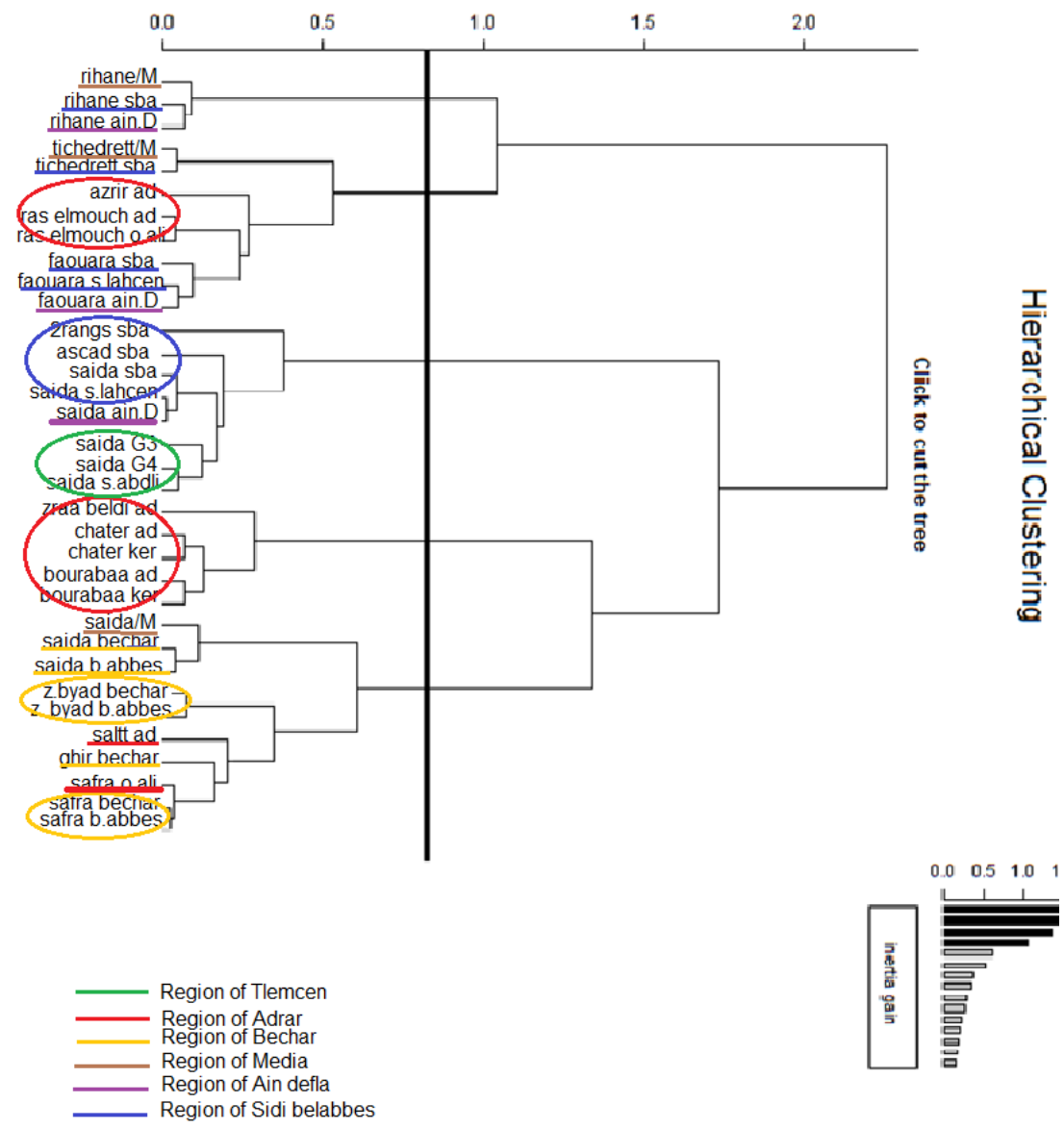

Figure. 5. Hierarchical Ascendante Classification (HAC) for qualitative characters of Barley in Algeria

Concerning the characters: Grain: hairiness of ventral furrow, Awn color, Ear attitude, ear shape, media spikelet lenght of glume and its awn relative tograin, sterile spikelete attitude(in mid-third of ear), indices $\left(\mathrm{H}^{\prime}=0.41\right)$, $\left(\mathrm{H}^{\prime}=0.44\right), \quad\left(\mathrm{H}^{\prime}=0.47\right)\left(\mathrm{H}^{\prime}=0.56\right)$ and $\left(\mathrm{H}^{\prime}=0.67\right)$ were found respectively.

Similar indices were registered for the characters: alignment of articles at the middle tier of ear(zigzag), Grain: hairiness of ventral furrow and Awns: anthocyanin coloration of tips with $\left(\mathrm{H}^{\prime}=0.53\right)$, and for ear: shape of the extremity and bosse of articles at the middle tier of ear with $\left(\mathrm{H}^{\prime}=0.58\right)$. Nearby indices were found for disposition of lodicules and curvature of first segment with $\left(\mathrm{H}^{\prime}=0.63\right)$ and $\left(\mathrm{H}^{\prime}=0.64\right)$ respectively.

The first PCA of the 12 quantitative characters explained $65.5 \%$ of the total variation among the 34 barley landraces (Figure 3). Characters related to yield (Number of grains per ear, density of grains per ear, ear density, ear width) presented the largest loadings on the first component, and the morphology, like Plant length, stem length, awn length, ear length, for the second component. The correlation of these characteristics may be explained by the influence of genes. These traits are controlled by a number of genes, and in general, they react in the same manner according to environmental conditions (Bellatreche et al.2017). In the second PCA of the 18 qualitative characters, the formation of three groups of characters is distinguished. This shows a positive correlation between the characters within the same groups and a negative correlation among the characters at the level of the $1 \mathrm{st}$ group (Beards color, kennel: color of aleurone layer, zigzag,and media spikelet length of glume and its awn relative to grain,and the parameters of the 3rd group(Grain: rachilla hair type, ear: shape of the extremity, Grain: hairiness of ventral furrow).

In this study, the two dendrograms for quantitative and qualitative characters show a relationship between different accessions.

We also notice, different accessions in the same region or $\mathrm{t}$ he same variety in different regions, that allows us to concl ude that there are two effects: 1)Environmental effect because we have the same variety in different regions as for Saida Tlemcen, Saida Sidi Belabbes, Saida Media, Saida Ain Defla and Saida Bechar for the quantitative characters, whereas for the qualitatives characters, we have Rihane Sidi Belabbes, Rihane Media and Rihane Ain Defla 
.2)Genetic effect because there are different accessions in the same region as Salt, Chater, Safra, Bourabaa, Zraa Beldi accessions in the region of Adrar for the quantitative characters. The hierarchical ascending classification (HAC) and the principal components analysis of the 12 recorded quantitative traits revealed consistent relationships within accessions. Similar results were found in barley by Molina and La Cruz del Campo (1977).

In conclusion, this work has shown that there is a high diversity due to the presence of high polymorphic characters (plant height, stem length, spike length with beard, spike length without beard, spike width, weight spike, length beards, number of seeds per spike, weight of seeds per spike). Finally, we want to stress that the use of these accessions in our breeding program has produced good results for semi-arid areas.

\section{REFERENCES}

Belaid A. 2000. Durum wheat in WANA (West Asia and North Africa): production, trade, and gains from technological change. In: Royo C, Nachit M, Di Fonzo N, Araus JL (eds.). Durum Wheat Improvement in the Mediterranean Region: New Challenges. CIHEAM-IAMZ, Zaragoza, Spain

Bellatreche A, Mahdad M Y, Kahouadji K, Gaouar SBS. 2017. Agromorphological diversity of some accessions of bread wheat (Triticum aestivum) in Western Algeria. BIodiversitas 18: 409-415. DOI: 10.13057/biodiv/d180153

Bellatreche A, Gaouar SBS. 2012. Preliminary genetic study of some varieties of durum wheat and bread wheat in the wilaya of Tlemcen and the influence of the environment on their yields. Sci Nat 4 (2): 37-42.

Bettaieb, B.L. and J. Attias. 1992. Electrophoretic heterogeneity of the hordeins of five barley (Hordeum vulgare L.) cultivars from Tunisia. C. R. Acad. Sci. Paris. t. 314, Série III: 141-146.

Boukhari Rachid and Suheil Samir Bachir Gaouar. 2018 Caractérisation de Quelques variétés d'olivier Algériennes (Tizi Ouzou). Editions Universitaires europeennes.Riga.lettonie. [France]

Ceccarelli S, Grando S. 1996. Importance of specific adaptation in breeding for marginal conditions. In Proc. of the first barley research review workshop, 16-19 October 1993, Addis Abeba: IAR/ ICARDA. Addis Abeba, Ethiopia.

Cross RJ. 1994. Geographical trends within a diverse spring barley collection as identified by agro/morphological and electrophoretic data. Theor Appl Genet 88: 597-603.

Dawson IK, Russel J, Powell W, Steffenson B, Thomas WTB, Waugh R. 2015.Barley: a translational model for adaptation to climate change. New Phytologist 206: 913-931

Dejene T, Andrea MB, Jens L .2010. Morphological diversity of Ethiopian barleys in relation to geographic regions and altitudes. Hereditas 147: 154-164.

Demissie A, and Bjqrnstad A .1996. Phenotypic diversity of Ethiopian barleys in relation to geographical regions, altitudinal range, and agro-ecological zones: as an aid to germplasm collection and conservation strategy. Hereditas 124: 17-29.
Gabillard D.1983: Amélioration du rendement protéique de l'orge: génétique quantitative et agro - physiologie. [Thèse de docteur de 3ème cycle]. sciences agronomiques. Institut National polytechnique de Toulouse, France. [France]

Gegnaw ST, Hadado, TT. 2014. Genetic diversity of qualitative traits of barley (Hordeum vulgare L.) landrace populations collected from Gamo Highlands of Ethiopia. Intl J Biodiv Conserv 6 (9): 663-673.

Hadjichristodoulou A. 1 995. Evaluation of barley landraces and selections from natural outcross of H.vulgare ssp. spontaneum with ssp. vulgare for breeding in semi-arid areas. Genet Resour Crop Evol 42: 83-89.

Harlan JR. 1975. Crops and Man, In: American Society of Agronomy and Crop Science Society of America, Madison, WI, USA.

Jaradat AA, Shahid M, Al Maskri AY. 2004.Genetic diversity in the Batini barley landrace from Oman: I. Spike and seed quantitative and qualitative traits. Crop Sci 44: 304-315

Lasa JM, Igartua E, Ciudad FJ, Codesal P, Garcia EV, Gracia MP, Medina B, Romagosa I, Molina-Cano JL, Montoya JL. 2001. Morphological and agronomical diversity patterns in the Spanish barley core collection. Hereditas 135: 217-225.

Mekonnon B, Lakew B, Dessalegn T. 2015. Morphological diversity and association of traits in Ethiopian food barley (Hordeum vulgare L.) landraces in relation to regions of origin and altitudes. J Plant Breed Crop Sci 7 (2): 44-54. DOI: 10.5897/JPBCS2014.0480

Mohamed S, Gaouar SBS. 2017. Diversité oléicole au niveau de la wilaya de Tlemcen. Editions universitaires européennes. [France]

Molina C, La Cruz del Campo JL .1977. Numerical taxonomy as an aid to barley germplasm collection. Barley Genet Newsl 7: 45-50

Negassa M. 1985. Patterns of phenotypic diversity in an Ethiopian barley collection, and the Arussi-Bale Highland as a center of origin of barley. Herediras 102: 139-150.

Papa R, Attene G, Barcaccia G, Ohgata A, Konishi T. 1998. Genetic diversity in landrace populations of Hordeum vulgare L. from Sardinia, Italy, as revealed by RAPDs, isozymes and morphophenological traits. Plant Breed 117: 523-530.

Rahal-Bouziane H, Alane F, Abdelguerfi A. 2015. Forage quality, forage dry matter yield, grain protein and agronomic traits of traditional barley genotypes (Hordeum vulgare L.) from rural areas in Algeria. Livestock Res Rural Dev 27: 182, http://www.lrrd.org/lrrd27/9/raha27182.html

Reid DA. 1985. Morphology and anatomy of the barley plant. In: Rasmusson RC. (eds.). Barley. ASSA, CSSA, SSSA, Madison, WI, USA.

Russell JR, Fuller J, Young G, Thomas B, Taramino G, Macaulay M, Waugh R, Powell W.1997. Discriminating between barley genotypes using microsatellite markers. Genome 40 (4): 442-450.

Shannon CE, Weaver W. 1948. The Mathematical Theory of Communication. University of Illinois Press, Urbana, IL.

UPOV [union internationale pour la protection des obtentions végétales].1988. Principes directeurs pour la conduite de l'examen des caractères distinctifs, del'homogénéité et de la stabilité, orge: 332. [France]

Yakoube K, Delloumi F, Bellatreche A. 2018. Morphometric characterization of the bread wheat variety "iskandaria" in the region of Aougrout (Adrar). Genet Biodiv J 2 (1): 12-18

Yassine M, Gaouar SBS. 2016 Le caroubier (Ceratonia siliqua L.) dans le Nord-Ouest de l'Algérie, situation et perspective d'amélioration. Editions universitaires européennes. [France]

Zeng XQ. 2015. Genetic variability in agronomic traits of a germplasm collection of hulless barley. Genet Mol Res 14 (4): 18356-18369.

Zhu YY, Chen HR, Fan JH, et al. 2000. Genetic diversity and disease control in rice. Nature 406: 718-722. 